\title{
The Relationship between Risk Factors of Metabolic Syndrome and Fatty Liver Detected Using Ultrasonography
}

\author{
Tadahiko Mitsumune'; Hiroyuki Shimomura²; Etsuo Senoh ${ }^{1}$; and Eizoh Kayashima ${ }^{1}$ \\ 'Junpukai Health Maintenance Center, and ${ }^{2}$ First Department of Internal Medicine, Okayama University
}

\begin{abstract}
Objectives To determine whether fatty liver is associated with risk factors of metabolic syndrome independently of body mass index (BMI).

Design A cross-sectional study.

Setting and Participants A total of 8,555 subjects, who consulted our institute for health screening between January 2001 and Decem-
\end{abstract} ber 2001.

Measurement and Results Among the risk factors of metabolic syndrome, overweight, hyperlipidemia, hyperglycemia, and hypertension, in that order, were significantly associated with fatty liver in multiple logistic regression analyses. The odds ratios (95\% Confidence Interval) were 6.3 (5.6-7.1), 2.4 (2.1-2.7), 1.8 (1.5-2.2), and 1.4 (1.2-1.6), respectively.

Fatty liver was significantly associated with an increase in the risk factors for metabolic syndrome. However, the increase in the odds ratios was rather limited on multivariate analyses when BMI was added compared to that on univariate analyses; nevertheless, subjects with 4 risk factors for metabolic syndrome had approximately 4 times (95\% Confidence Interval: 2.1-6.5) the risk of fatty liver compared with that of those without any risk factors.

BMI increased the odds ratios of having 2 or more risk factors for metabolic syndrome in subjects both with and without fatty liver. Subjects with fatty liver had approximately 2 times the risk of having 2 or more risk factors compared with that of those without fatty liver in each BMI group.

Conclusions These results suggest that individuals with fatty liver, even if they were not overweight, are probably complicated by the risk of metabolic syndrome, that is, a fatty liver per se also has clinical significance. However, since these pathological findings were also dependent on BMI, we believe that it is possible to reduce the risk of metabolic syndrome by reducing body weight, even if a person has a fatty liver.

Key Words Fatty Liver; Metabolic Syndrome; Obesity; Ultrasonography; Multiple Logistic Regression Analysis

\section{INTRODUCTION}

Recently, metabolic syndrome, which involves several risk factors including obesity, hypertension, hyperlipidemia, and glucose intolerance, has been noted as an important foundation of coronary heart disease. ${ }^{[1]-[5]}$ Non-alcoholic fatty liver, a condition characterized by excess accumulation of lipids within hepatocytes, is generally thought to have a benign process with a relatively small risk of progression to advanced disease. ${ }^{[6]}$ A finding of fatty liver has often been detected on abdominal ultrasonography in health screening, and is likely to be accompanied by overweight. However, fatty liver may also be associated with metabolic syndrome

Received: April 1, 2003

'Address; Junpukai Health Maintenance Center, 2-3-1 Daiku, Okayama, 700-0913 Japan. TEL: 81-86-226-2666, FAX: 81-86-226-0370, E-mail: JBH01210@nifty.ne.jp

${ }^{2}$ Address; First Department of Internal Medicine, Okayama University, 2-5-1 Shikata-cho, Okayama, 700-8558 Japan. TEL: 81-86-235-7219, FAX: 81-86-225-5991, E-mail: hshimomu@md.okayama-u.ac.jp independently of obesity.

To determine whether fatty liver is associated with risk factors of metabolic syndrome independently of body mass index (BMI), we performed a cross-sectional study using multivariate analyses adjusted for BMI.

\section{MATERIALS AND METHODS}

A total of 8,555 subjects (6,136 males, 2,419 females), who consulted our institute for health screening between January 2001 and December 2001, were enrolled in this study.

Each risk of metabolic syndrome was defined as follows; (1) overweight: BMI $\geqq 25.0 \mathrm{~kg} / \mathrm{m}^{2}$, (2) hypertension: blood pressure $\geqq 140 / 90 \mathrm{mmHg}$, or under a doctor's care for hypertension, (3) hyperglycemia: fasting plasma glucose $\geqq 126 \mathrm{mg} / \mathrm{d} l$, or under a doctor's care for diabetes, (4) hyperlipidemia: serum total cholesterol $\geqq 220 \mathrm{mg} / \mathrm{d} l$, triglycerides $\geqq 150 \mathrm{mg} / \mathrm{d} l$, high density lipoprotein cholesterol, $<40 \mathrm{mg} / \mathrm{d} l$, low density lipoprotein cholesterol $\geqq 140 \mathrm{mg} / \mathrm{d} l$, or under a doctor's care for hyperlipidemia.

BMI was classified into 5 groups by quintile.

The diagnosis of fatty liver was based on liver/kidney contrast, deep attenuation, and vascular blurring on ultrasonography. The examiner was blinded to the results of laboratory tests.

\section{Statistics}

Univariate chi-square test was used to estimate the crude odds ratios. A multiple logistic regression model incorporating 4 risk factors of metabolic syndrome, age, gender, and smoking habit was constructed. The odds ratios were calculated as the ratios of the odds of having fatty liver in subjects with each risk factor compared to subjects without any risk factors. A second model incorporating each number of risk factors of metabolic syndrome, age, gender, smoking habit, and BMI was constructed. Odds ratios were calculated as the ratio of the odds of having fatty liver in subjects with risk factors of metabolic syndrome compared to that in subjects without any risk factors. A third model incorporating each BMI group with or without fatty liver, age, gender, and smoking habit was constructed. Odds ratios were calculated as the ratio of the odds of having 2 or more risk factors of metabolic syndrome in subjects of each BMI group compared to subjects of group 1 without fatty liver.

Previous smokers were counted as having a negative smoking habit.

All statistical analyses were performed using SPSS for Windows. All tests were interpreted using a significance level of $p<$ 0.05 .

\section{RESULTS}

The mean age of the subject population was $48.9 \pm 8.9$ years. Of the 8,555 subjects, $6,136(71.7 \%)$ were male. 
Prevalence of fatty liver was increased according to increasing BMI. In group 5 (BMI $\geqq 25.40$ ), more than $60 \%$ of subjects demonstrated fatty liver, while less than $5 \%$ of subjects had those in group 1 (BMI < 20.42) showed such findings (Table 1).

In relation to fatty liver, each risk factor of metabolic syndrome, overweight, hyperlipidemia, hyperglycemia, and hypertension, in that order, was significantly associated with fatty liver on multiple logistic regression analyses. The odds ratios $(95 \%$ Confidence Interval) were 6.3 (5.6-7.1), 2.4 (2.1-2.7), 1.8 (1.5-2.23), and 1.4
(1.2-1.6), respectively (Table 2).

Fatty liver was significantly associated with an increase in risk factors of metabolic syndrome. However, the increase of odds ratios was rather limited on multivariate analysis when BMI was added than by either univariate analysis or age-gender-smokingadjusted analysis; nevertheless, subjects with 4 risk factors of metabolic syndrome had approximately 4 times (95\% Confidence Interval: 2.1-6.5) the risk of fatty liver compared with that of those without any risk factors (Table 3 ).

Table 1 Distribution of subjects with fatty livers in each body mass index group.

\begin{tabular}{lcrrrr}
\hline \multirow{2}{*}{ BMI group } & \multirow{2}{*}{ BMI } & $\mathrm{N}\left(\%^{\mathrm{a}}\right)$ & \multicolumn{1}{c}{ Male } & & Female \\
\cline { 5 - 5 } & & & $\mathrm{N}\left(\%^{\mathrm{a}}\right)$ & & $\mathrm{N}\left(\%^{\mathrm{a}}\right)$ \\
\hline Group 1 $(n=1,711)$ & $<20.42$ & $63(3.7)$ & $42(4.8)$ & & $21(2.5)$ \\
Group 2 $(n=1,711)$ & $20.42-22.08$ & $192(11.2)$ & $152(13.2)$ & & $40(7.1)$ \\
Group 3 $(n=1,711)$ & $22.09-23.59$ & $379(22.2)$ & $323(25.4)$ & & $56(12.8)$ \\
Group 4 $(n=1,711)$ & $23.60-25.39$ & $659(38.5)$ & $576(41.0)$ & $83(27.1)$ \\
Group 5 $(n=1,711)$ & $\geqq 25.40$ & $1,133(66.2)$ & $962(67.3)$ & & $171(61.3)$ \\
Total $(n=8,555)$ & & $2,426(28.4)$ & $2,055(33.5)$ & $371(15.3)$ \\
\hline
\end{tabular}

BMI: Body Mass Index

OR: odds ratio

${ }^{\mathrm{a}}$ : Percentage of subjects with fatty livers in each BMI group

$\mathrm{N}$ : Number of fatty liver

Table 2 Odds ratios of fatty liver with each risk factor of metabolic syndrome.

\begin{tabular}{ccc}
\hline Risk Factor & $\begin{array}{c}\text { Crude OR } \\
(95 \% \mathrm{CI})\end{array}$ & $\begin{array}{c}\text { Multivariate OR } \\
(95 \% \mathrm{CI})\end{array}$ \\
\hline 0 & 1.0 & 1.0 \\
Hypertension & $1.4(1.2-1.6)$ & $1.4(1.2-1.6)$ \\
Hyperglucosemia & $1.9(1.5-2.3)$ & $1.8(1.5-2.2)$ \\
Hyperlipidemia & $2.4(2.2-2.7)$ & $2.4(2.1-2.7)$ \\
Overweight & $6.8(6.0-7.6)$ & $6.3(5.6-7.1)$ \\
\hline
\end{tabular}

OR: odds ratio

CI: confidence interval

Odds ratios refer to the risk of having fatty liver in subjects with each risk factor compared to subjects without any risk factors. Multivariate OR was adjusted for age, gender, and smoking habit.
Table 3 Odds ratios of fatty liver with risk factors of metabolic syndrome.

\begin{tabular}{cccc}
\hline No. of risk factor & $\begin{array}{c}\text { Crude OR } \\
(95 \% \mathrm{CI})\end{array}$ & $\begin{array}{c}\text { Adjusted OR } \\
(95 \% \mathrm{CI})\end{array}$ & $\begin{array}{c}\text { Multivariate OR } \\
(95 \% \mathrm{CI})\end{array}$ \\
\hline 0 & 1.0 & 1.0 & 1.0 \\
1 & $3.3(2.8-3.8)$ & $3.4(2.9-3.9)$ & $2.1(1.7-2.4)$ \\
2 & $10(8.5-12)$ & $10(8.4-12)$ & $2.7(2.5-3.3)$ \\
3 & $22(17-28)$ & $23(18-29)$ & $3.8(2.9-5.0)$ \\
4 & $26(15-44)$ & $26(15-45)$ & $3.7(2.1-6.5)$ \\
\hline
\end{tabular}

OR: odds ratio

CI: confidence interval

Odds ratios refer to the risk of having fatty liver in subjects with risk factors of metabolic syndrome compared to subjects without any risk factors.

Adjusted OR was adjusted for age, gender, and smoking habit.

Multivariate OR was adjusted for age, gender, smoking habit, and body mass index.

Table 4 Odds ratios of having 2 or more risk factors of metabolic syndrome in persons with and without fatty liver, according to BMI.

\begin{tabular}{lcccccc}
\hline \multirow{2}{*}{ BMI group } & \multirow{2}{*}{ BMI } & \multicolumn{2}{c}{ Non-fatty liver } & & \multicolumn{2}{c}{ Fatty liver } \\
\cline { 3 - 4 } \cline { 6 - 7 } & & $\begin{array}{c}\text { Crude OR } \\
(95 \% \mathrm{CI})\end{array}$ & $\begin{array}{c}\text { Multivariate OR } \\
(95 \% \mathrm{CI})\end{array}$ & & $\begin{array}{c}\text { Crude OR } \\
(95 \% \mathrm{CI})\end{array}$ & $\begin{array}{c}\text { Multivariate OR } \\
(95 \% \mathrm{CI})\end{array}$ \\
\hline Group 1 $(n=1,711)$ & $<20.42$ & 1.0 & 1.0 & & $2.4(1.1-5.4)$ & $2.5(1.1-5.6)$ \\
Group 2 $(n=1,711)$ & $20.42-22.08$ & $1.7(1.3-2.3)$ & $1.5(1.1-2.0)$ & & $4.0(2.6-6.1)$ & $3.3(2.1-5.1)$ \\
Group 3 $(n=1,711)$ & $22.09-23.59$ & $2.4(1.8-3.1)$ & $1.9(1.4-2.6)$ & & $4.6(3.2-6.4)$ & $3.6(2.5-5.1)$ \\
Group 4 $(n=1,711)$ & $23.60-25.39$ & $5.6(4.3-7.3)$ & $4.6(3.5-6.0)$ & & $10(7.1-13)$ & $8.3(6.3-11)$ \\
Group 5 $(n=1,711)$ & $\geqq 25.40$ & $58(43-77)$ & $55(40-74)$ & & $100(76-131)$ & $107(80-142)$ \\
\hline
\end{tabular}

BMI: Body Mass Index

OR: odds ratio

CI: confidence interval

Odds ratios refer to the risk of having 2 or more risk factors of metabolic syndrome in subjects of each BMI group compared to subjects of group 1 without fatty liver.

Multivariate OR was adjusted for age, gender, and smoking habit. 
BMI increased the odds ratios of having 2 or more risk factors of metabolic syndrome in subjects either with or without fatty liver. Subjects with fatty liver had approximately 2 times the risk of having 2 or more risk factors compared with those without fatty liver in each BMI group (Table 4).

\section{DISCUSSION}

Fatty liver should be diagnosed by liver biopsy. However, liver biopsies are now rarely performed because ultrasonic diagnosis has been established. ${ }^{[7], ~[8]}$ The main diagnostic criteria for ultrasonic diagnosis of fatty liver are liver/kidney contrast, deep attenuation, and/or vascular blurring, and we adopted all of these 3 factors as parameters for diagnosing fatty liver in this series. The incidence and distribution of fatty liver in our investigation were compatible with a previous report in Japan, ${ }^{[9]}$ therefore, we consider that the diagnoses were appropriate.

It has recently been shown that insulin resistance is related to the cause of fatty liver, as well as metabolic syndrome. The increased basal expression of tumor necrosis factor- $\alpha$ (TNF- $\alpha$ ) by adipose tissue in obese people has been proposed to be an important mechanism for limiting obesity by increasing insulin resistance. ${ }^{[10]}$ However, we could not refer to this because plasma insulin and/or TNF- $\alpha$ were not examined in this study. It will probably be necessary to conduct studies using an examination of insulin resistance.

Recently, genetic factors in lifestyle-related diseases have included single nucleotide polymorphisms (SNPs). ${ }^{[1]}$ SNPs, including beta-adrenergic receptor, uncoupling proteins, calpain 10 , and leptin receptor are also responsible for obesity. The Rtype SNP of the beta-adrenergic receptor is known to be a predisposing factor for obesity caused by westernization of Japanese dietary habits. ${ }^{[11]}$ However, it is unclear whether normal weight individuals with fatty livers corresponded to this type because there was no examination of SNPs in this study. We expect SNPs to be easily and widely applied in health screening to prevent metabolic syndrome.

In the present study, since an increase in the odds ratio was rather limited on multivariate analysis when BMI was added compared to that on univariate analysis or age-gender-smoking-adjusted analysis, BMI might be a confounding factor. We should adjust for BMI if fatty liver is used as a variable.

In conclusion, the present results suggest that individuals with fatty liver, even if they are not overweight, are probably complicated by the risk of metabolic syndrome, that is, a fatty liver per se also has clinical significance. However, since these pathological findings were also dependent on BMI, we conclude that it is possible to reduce the risk of metabolic syndrome by reducing body weight, even if the person has a fatty liver. Therefore, individuals with fatty liver detected by ultrasonography should be actively guided in dietary modification and exercise.

\section{REFERENCES}

[1] Larkin, M.: "Metabolic Syndrome" Targeted in New US Cholesterol Guidelines, Lancet, 357: 1594, 2001

[2] Ford, E.S., Giles, W.H., Dietz, W.H.: Prevalence of the Metabolic Syndrome among US Adults: Findings from the Third National Health and Nutrition Examination Survey, JAMA, 287: 356-359, 2002

[3] Nakamura, T., Tsubono, Y., Kameda-Takemura, K., Funahashi, T., Yamashita, S., Hisamichi, S., Kita, T., Yamamura, T., Matsuzawa, Y.: Magnitude of Sustained Multiple Risk Factors for Ischemic Heart Disease in Japanese Employees-A Case-control Study-, Jpn Circ J, 65: 11-17, 2001

[4] Björntorp, P., Rosmond, R.: The Metabolic Syndrome-A Neuroendocrine Disorder? Br J Nutr, 83 Suppl 1: S49-S57, 2000

[5] National Cholesterol Education Program: Executive Summary of the Third Report of the National Cholesterol Education Program (NCEP) Expert Panel on Detection, Evaluation, and Treatment of High Blood Cholesterol in Adults (Adult Treatment Panel III), JAMA, 285: 2486-2497, 2001

[6] Fong, D.G., Nehra, V., Lindor, K.D., Buchman, A.L.: Metabolic and Nutritional Considerations in Non-alcoholic Fatty Liver, Hepatology, 32: 3-10, 2000

[7] Joseph, A.E.A., Dewbury, K.C., McGuire, P.G.: Ultrasound in Detection of Chronic Liver Disease (The "Bright Liver"), Br J Radiol, 52: 184, 1986

[8] Yajima, Y., Ohta, K., Narui, T., Abe, R., Suzuki, H., Ohtsuki, M.: Ultrasound in the Diagnosis of Fatty Liver, Significance of the Liver/Kidney Contrast, Acta Hepatologica Japonica, 23: 903-908, 1982 (in Japanese)

[9] Kamoshita, T., Hoshi, K., Ohe, T., Yoshida, S., Nakajima, Y., Toyoda, Y., Satukawa, K.: Examination of Bright Liver with Abdominal Ultrasonography in Human Dry Dock, Official Journal of the Japanese Society of Human Dry Dock, 11: 244-248, 1996 (in Japanese)

[10] James, O., Day, C.: Non-alcoholic Steatohepatitis: Another Disease of Affluence, Lancet, 353: 1634-1636, 1999

[11] Kagawa, Y.: Nutrition and Genetics in the Prevention of Lifestyle Related Disease, Health Evaluation and Promotion, 29: 613-617, 2002 (in Japanese) 